\title{
Affine descents and the Steinberg torus
}

\author{
Kevin Dilks, T. Kyle Petersen and John R. Stembridge $\|^{\dagger}$ \\ Department of Mathematics, University of Michigan, Ann Arbor MI 48109-1043
}

\begin{abstract}
Let $W \ltimes L$ be an irreducible affine Weyl group with Coxeter complex $\Sigma$, where $W$ denotes the associated finite Weyl group and $L$ the translation subgroup. The Steinberg torus is the Boolean cell complex obtained by taking the quotient of $\Sigma$ by the lattice $L$. We show that the ordinary and flag $h$-polynomials of the Steinberg torus (with the empty face deleted) are generating functions over $W$ for a descent-like statistic first studied by Cellini. We also show that the ordinary $h$-polynomial has a nonnegative $\gamma$-vector, and hence, symmetric and unimodal coefficients. In the classical cases, we also provide expansions, identities, and generating functions for the $h$-polynomials of Steinberg tori.

Résumé. Nous considérons un groupe de Weyl affine irréductible $W \ltimes L$ avec complexe de Coxeter $\Sigma$, où $W$ désigne le groupe de Weyl fini associé et $L$ le sous-groupe des translations. Le tore de Steinberg est le complexe cellulaire Booléen obtenu comme le quotient de $\Sigma$ par $L$. Nous montrons que les $h$-polynômes, ordinaires et de drapeaux, du tore de Steinberg (sans la face vide) sont des fonctions génératrices sur $W$ pour une statistique de type descente, étudiée en premier lieu par Cellini. Nous montrons également qu'un $h$-polynôme ordinaire possède un $\gamma$-vecteur positif, et par conséquent, a des coéfficients symétriques et unimodaux. Dans les cas classiques, nous donnons également des développements, des identités et des fonctions génératrices pour les $h$-polynômes des tores de Steinberg.
\end{abstract}

Keywords: Coxeter complex, $\gamma$-nonnegativity, descent statistic, Eulerian polynomial

\section{Introduction}

Let $S_{n}$ denote the symmetric group of permutations of $[n]:=\{1, \ldots, n\}$. For each $w \in S_{n}$, a descent is an index $i(1 \leqslant i<n)$ such that $w_{i}>w_{i+1}$. We let

$$
d(w):=\left|\left\{i \in[n-1]: w_{i}>w_{i+1}\right\}\right|
$$

denote the number of descents in $w$. The corresponding generating function

$$
A_{n-1}(t):=\sum_{w \in S_{n}} t^{d(w)}
$$

is known as an Eulerian polynomial, although this definition differs from the classical one by a power of $t$. Some interesting features of the Eulerian polynomials include the facts that they have symmetric and unimodal coefficients and are known to have all real roots.

\footnotetext{
${ }^{\dagger}$ The work of the third author was supported in part by an NSA Grant. 
More generally, if $W$ is any finite Coxeter group with simple reflections $s_{1}, \ldots, s_{n}$ (such as the symmetric group $S_{n+1}$ with simple transpositions $s_{i}=(i, i+1)$ ), then a descent in some $w \in W$ may be defined as an index $i$ such that $\ell\left(w s_{i}\right)<\ell(w)$, where $\ell(w)$ denotes the minimum length of an expression for $w$ as a product of simple reflections. Thus there is an analogous $W$-Eulerian polynomial

$$
W(t):=\sum_{w \in W} t^{d(w)},
$$

where $d(w)$ is defined to be the number of descents in $w$. Note that as a Coxeter group, $S_{n}$ is often denoted $A_{n-1}$, so this notation is consistent with (1.1).

Like the classical Eulerian polynomials, the $W$-Eulerian polynomials are known to have symmetric and unimodal coefficients. An elegant explanation of this fact may be based on a topological interpretation of $W(t)$ as the $h$-polynomial of the Coxeter complex of $W$. Since every (finite) Coxeter complex is realizable as the boundary complex of a simplicial polytope, the symmetry and unimodality of the coefficients of $W(t)$ may thus be seen as a consequence of the $g$-theorem (e.g., see Section III.1 of (21)).

Recently, several authors (see for example $(1,13,20,26)$ ) have identified interesting classes of simplicial complexes whose $h$-polynomials have expansions of the form

$$
h(t)=\sum_{0 \leqslant i \leqslant n / 2} \gamma_{i} t^{i}(1+t)^{n-2 i},
$$

where the coefficients $\gamma_{i}$ are nonnegative. It is easy to see that each summand in this expansion has symmetric and unimodal coefficients centered at $n / 2$, and thus any $h$-polynomial with a nonnegative " $\gamma$-vector" in this sense necessarily has symmetric and unimodal coefficients. In these terms, the $h$ polynomials of all finite Coxeter complexes (i.e., the $W$-Eulerian polynomials) are known to have nonnegative $\gamma$-vectors (26).

Another feature of $\gamma$-nonnegativity is that it is a necessary condition for a polynomial to have all real roots, given that the polynomial has nonnegative symmetric coefficients. In this direction, Brenti (2) has conjectured that the $W$-Eulerian polynomials have all real roots, a result that remains unproved only for the groups $W=D_{n}$.

In this paper, we study a family of Eulerian-like polynomials associated to irreducible affine Weyl groups. These "affine" Eulerian polynomials may be defined as generating functions for "affine descents" over the corresponding finite Weyl group. An affine descent is similar to an ordinary descent in a Weyl group, except that the reflection corresponding to the highest root may also contribute a descent, depending on its effect on length.

The affine Eulerian polynomials have a number of interesting properties similar to those of the ordinary $W$-Eulerian polynomials. In particular, we show that they have nonnegative $\gamma$-vectors (Theorem 4.2], and conjecture that all of their roots are real. Perhaps the most interesting similarity is that each affine Eulerian polynomial is the $h$-polynomial of a naturally associated relative cell complex (Theorem 3.1).

To describe this complex, one should start with an irreducible affine Coxeter arrangement. Such an arrangement induces a simplicial decomposition of the ambient space; by taking the quotient of this space by the translation subgroup of the associated affine Weyl group, one obtains a torus decomposed into simplicial cells. We refer to this cell complex as the Steinberg torus in recognition of the work of Steinberg, who gave a beautiful proof of Bott's formula for the Poincaré series of an affine Weyl group by analyzing the action of the finite Weyl group on the homology of this complex in two different ways (see 
Section 3 of (23)). In fact, Steinberg also allows the possibility of twisting the entire construction by an automorphism, but we will not consider this variation here.

It is important to note that the Steinberg torus is not a simplicial complex (distinct cells may share the same set of vertices), but it is at least a Boolean cell complex in the sense that all lower intervals in the partial ordering of cells are Boolean algebras ${ }^{(i)}$ For further information about Boolean complexes, see (22) and the references cited there.

For our purposes, it is essential to omit the empty cell of dimension -1 from the Steinberg torus; we refer to the resulting relative complex as the reduced Steinberg torus. It is this complex whose $h$-polynomial is the corresponding affine Eulerian polynomial; i.e., the generating function for affine descents.

It is noteworthy that affine descents in finite Weyl groups were first introduced by Cellini (3) in a construction of a variant of Solomon's descent algebra, and developed further for the groups of type $A$ and $C$ in several follow-up papers on "cyclic descents" by Cellini (4; 5), Fulman (11; 12), and Petersen (18). In very recent work, Lam and Postnikov (16) study a weighted count of affine descents (the "circular descent number") that coincides with an ordinary count (only) in type $A$.

The paper is structured as follows. Section 2 introduces the necessary definitions, including details of the construction of the Steinberg torus. In Section 3 we show that the affine Eulerian polynomials are the $h$-polynomials of reduced Steinberg tori (Theorem 3.1). We also show that reduced Steinberg tori are partitionable (Remark 3.6); this is a weak analogue of shellability that implies $h$-nonnegativity. It is also noteworthy that this nonnegativity is implied by very recent work of Novik and Swartz (17) from which it follows that a reduced Boolean cell complex homeomorphic to a torus will indeed have a nonnegative $h$-vector (27).

In Section 4, we present our second main result; namely, that the affine Eulerian polynomials have nonnegative $\gamma$-vectors (Theorem 4.2). As a corollary, it follows that the $h$-vectors of reduced Steinberg tori are symmetric and unimodal. In this section, we also present evidence supporting our conjecture that all roots of affine Eulerian polynomials are real. The proof of Theorem 4.2 is case-by-case, and relies on combinatorial expansions for the $\gamma$-vectors of affine Eulerian polynomials for the classical Weyl groups that we provide in Section 5 .

In Section 6, we present three unexpected identities relating ordinary and affine Eulerian polynomials (two new, one old), and use these to derive exponential generating functions for the affine Eulerian polynomials for each classical series of Weyl groups.

This paper is an abridged version of $(8)$, which contains proofs of all the results presented here (and more).

\section{Preliminaries}

\subsection{Finite and affine Weyl groups}

We assume the reader is familiar with the basic theory of reflection groups. We follow the notational conventions of (15).

Let $\Phi$ be a crystallographic root system embedded in a real Euclidean space $V$ with inner product $\langle\cdot, \cdot\rangle$. For any root $\beta \in \Phi$, let $H_{\beta}:=\{\lambda \in V:\langle\lambda, \beta\rangle=0\}$ be the hyperplane orthogonal to $\beta$ and let $s_{\beta}$ denote the orthogonal reflection through $H_{\beta}$. Fix a set of simple roots $\Delta=\left\{\alpha_{1}, \ldots, \alpha_{n}\right\} \subset \Phi$, and let

(i) We thank V. Welker for bringing this to our attention. 
$S=\left\{s_{1}, \ldots, s_{n}\right\}$ denote the corresponding set of simple reflections. The latter generates a finite Coxeter group $W$ (a Weyl group).

Unless stated otherwise, we always assume that $\Phi$ and $W$ are irreducible.

For convenience, we assume that $\Delta$ spans $V$.

Having fixed a choice of simple roots, every root $\beta$ either belongs to the nonnegative span of the simple roots and is designated positive, or else belongs to the nonpositive span of the simple roots and is designated negative. We write $\beta>0$ or $\beta<0$ accordingly.

The affine Weyl group $\widetilde{W}$ is generated by reflections $s_{\beta, k}$ through the affine hyperplanes

$$
H_{\beta, k}:=\{\lambda \in V:\langle\lambda, \beta\rangle=k\} \quad(\beta \in \Phi, k \in \mathbb{Z}) .
$$

Alternatively, one may construct $\widetilde{W}$ as the semidirect product $W \ltimes \mathbb{Z} \Phi^{\vee}$, where $\mathbb{Z} \Phi^{\vee}$ denotes the lattice generated by all co-roots $\beta^{\vee}=2 \beta /\langle\beta, \beta\rangle(\beta \in \Phi)$, acting on $V$ via translations.

Given that $\Phi$ is irreducible, it has a unique highest root $\widetilde{\alpha}$, and it is well-known that $\widetilde{W}$ is generated by $\widetilde{S}:=S \cup\left\{s_{\widetilde{\alpha}, 1}\right\}$ and that $(\widetilde{W}, \widetilde{S})$ is an irreducible Coxeter system.

Note that $\widetilde{W}$ depends on the underlying root system $\Phi$ (not merely $W$ ), so we are committing an abuse of notation. For example, $B_{n}$ and $C_{n}$ are isomorphic as Coxeter systems, but the affine groups $\widetilde{B}_{n}$ and $\widetilde{C}_{n}$ are not isomorphic as Coxeter systems for $n \geqslant 3$.

\subsection{Coxeter complexes}

The hyperplanes $H_{\beta}(\beta \in \Phi)$ induce a partition of $V$ into a complete $W$-symmetric fan of simplicial cones. By intersecting this fan with the unit sphere in $V$, one obtains a topological realization of the Coxeter complex $\Sigma(W)$. The action of $W$ on chambers (maximal cones) in the fan is simply transitive, and the choice of simple roots $\Delta$ is equivalent to designating a dominant chamber; namely,

$$
C_{\varnothing}:=\{\lambda \in V:\langle\lambda, \alpha\rangle>0 \text { for all } \alpha \in \Delta\} .
$$

The closure of the dominant chamber is a fundamental domain for the action of $W$ on $V$, and thus every cone in the fan has the form $w C_{J}(w \in W, J \subseteq[n])$, where

$$
C_{J}:=\left\{\lambda \in V:\left\langle\lambda, \alpha_{j}\right\rangle=0 \text { for } j \in J,\left\langle\lambda, \alpha_{j}\right\rangle>0 \text { for } j \in[n] \backslash J\right\} .
$$

Notice that the rays (1-dimensional cones) have the form $w C_{J}$ where $J=[n] \backslash\{j\}$ for some $j$. If we assign color $j$ to all such rays, we obtain a balanced coloring of $\Sigma(W)$; i.e., every maximal face (chamber) has exactly one vertex (extreme ray) of each color.

Similarly, the affine hyperplanes $H_{\beta, k}(\beta \in \Phi, k \in \mathbb{Z})$ may be used to partition $V$ into a $\widetilde{W}$-symmetric simplicial complex that is isomorphic to the Coxeter complex $\Sigma(\widetilde{W})$. By abuse of notation, we will identify $\Sigma(\widetilde{W})$ with this particular geometric realization. The action of $\widetilde{W}$ on alcoves (maximal simplices) is simply transitive, and the fundamental alcove

$$
A_{\varnothing}:=C_{\varnothing} \cap\{\lambda \in V:\langle\lambda, \widetilde{\alpha}\rangle<1\}
$$

is tied to the choice of $\widetilde{S}$ in the sense that the $\widetilde{W}$-stabilizer of every point in the closure of $A_{\varnothing}$ (a fundamental domain) is generated by a proper subset of $\widetilde{S}$. We index the faces of $A_{\varnothing}$ by subsets of 
$[0, n]:=\{0,1, \ldots, n\}$ so that the $J$-th face is

$$
A_{J}:=\left\{\begin{aligned}
C_{J} \cap\{\lambda \in V:\langle\lambda, \widetilde{\alpha}\rangle<1\} & \text { if } 0 \notin J, \\
C_{J \backslash\{0\}} \cap\{\lambda \in V:\langle\lambda, \widetilde{\alpha}\rangle=1\} & \text { if } 0 \in J .
\end{aligned}\right.
$$

Note that $A_{J}$ is the empty face when $J=[0, n]$.

Since the closure of $A_{\varnothing}$ is a fundamental domain for the action of $\widetilde{W}$, each cell in this complex has the form $\mu+w A_{J}\left(\mu \in \mathbb{Z} \Phi^{\vee}, w \in W, J \subseteq[0, n]\right)$. In particular, the vertices of $\Sigma(\widetilde{W})$ are of the form $\mu+w A_{\{j\}^{c}}$, where $J^{c}:=[0, n] \backslash J$. If we assign color $j$ to each of the vertices $\mu+w A_{\{j\}^{c}}$, then the vertices of the cell $\mu+w A_{J}$ are assigned color-set $J^{c}$ (without repetitions), so this coloring is balanced.

Remark 2.1 If $\Phi$ and $W$ are reducible, then the affine hyperplanes $H_{\beta, k}$ may still be used to partition $V$ into a cell complex, but the result is not a geometric realization of the Coxeter complex of $\widetilde{W}$. Indeed, the cells of this complex are products of simplices, whereas the Coxeter complex of every Coxeter system is simplicial.

\subsection{Flag $f$-vectors and $h$-vectors}

Let $\Sigma$ be a finite set of simplices (or abstractly, a hypergraph) that is properly colored; i.e., the vertices of $\Sigma$ have been assigned colors from some index set, say $[0, n]$, so that no simplex has two vertices with the same color. What we have in mind here are balanced simplicial (or more generally, Boolean) complexes.

A basic combinatorial invariant of $\Sigma$ that carries significant algebraic and topological information (e.g., see the discussion in Section III.4 of (21) $)$ is the flag $h$-vector. The components of the flag $h$-vector are the quantities

$$
h_{J}(\Sigma):=\sum_{I \subseteq J}(-1)^{|J \backslash I|} f_{I}(\Sigma) \quad(J \subseteq[0, n]),
$$

where $f_{I}(\Sigma)$ denotes the number of simplices in $\Sigma$ whose vertices have color-set $I$.

The quantities $f_{J}(\Sigma)$ for $J \subseteq[0, n]$ are collectively referred to as the flag $f$-vector of $\Sigma$.

The corresponding generating functions

$$
\begin{aligned}
f\left(\Sigma ; t_{0}, \ldots, t_{n}\right) & :=\sum_{J \subseteq[0, n]} f_{J}(\Sigma) \prod_{j \in J} t_{j}, \\
h\left(\Sigma ; t_{0}, \ldots, t_{n}\right) & :=\sum_{J \subseteq[0, n]} h_{J}(\Sigma) \prod_{j \in J} t_{j}
\end{aligned}
$$

are known as the $f$ lag $f$-polynomial and flag $h$-polynomial of $\Sigma$. The more familiar ordinary $f$-polynomial and $h$-polynomial may be obtained via the specializations

$$
\begin{aligned}
& f(\Sigma ; t):=f(\Sigma ; t, \ldots, t)=\sum_{J \subseteq[0, n]} f_{J}(\Sigma) t^{|J|}, \\
& h(\Sigma ; t):=h(\Sigma ; t, \ldots, t)=\sum_{J \subseteq[0, n]} h_{J}(\Sigma) t^{|J|} .
\end{aligned}
$$

The coefficients of these polynomials yield the (ordinary) $f$-vector and $h$-vector of $\Sigma$. 

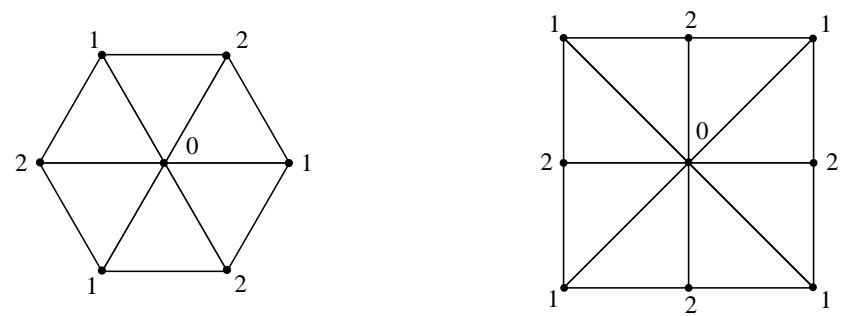

Fig. 1: The Steinberg tori for $\widetilde{A}_{2}$ and $\widetilde{C}_{2}$.

Note that 2.1) implies

$$
h\left(\Sigma ; t_{0}, \ldots, t_{n}\right)=\left(1-t_{0}\right) \cdots\left(1-t_{n}\right) f\left(\Sigma ; \frac{t_{0}}{1-t_{0}}, \ldots, \frac{t_{n}}{1-t_{n}}\right),
$$

and hence $h(\Sigma ; t)=(1-t)^{n+1} f(\Sigma ; t /(1-t))$.

\subsection{The Steinberg torus}

As the translation subgroup of $\widetilde{W}$, the co-root lattice $\mathbb{Z} \Phi^{\vee}$ acts as a group of color-preserving automorphisms of the affine Coxeter complex $\Sigma(\widetilde{W})$. Letting $T$ denote the $n$-torus $V / \mathbb{Z} \Phi^{\vee}$, it follows that the image of $\Sigma(\widetilde{W})$ under the natural map $V \rightarrow T$ is a balanced Boolean complex, denoted $\Sigma_{T}(\widetilde{W})$. That is,

$$
\Sigma_{T}(\widetilde{W})=\Sigma(\widetilde{W}) / \mathbb{Z} \Phi^{\vee}
$$

As explained in the introduction, we refer to $\Sigma_{T}(\widetilde{W})$ as the Steinberg torus. We also define the reduced Steinberg torus, denoted $\Sigma_{T}^{\prime}(\widetilde{W})$, to be the relative complex obtained by deleting the empty simplex of dimension -1 from $\Sigma_{T}(\widetilde{W})$.

Note that these are finite complexes; there is one maximal cell $w A_{\varnothing}+\mathbb{Z} \Phi^{\vee}$ for each $w \in W$.

There is an alternative way to construct the Steinberg torus that starts with the observation that the 0colored vertices in $\Sigma(\widetilde{W})$ are the members of $\mathbb{Z} \Phi^{\vee}$. Since every alcove $A$ has a unique 0 -colored vertex, one may translate $A$ via $\mathbb{Z} \Phi^{\vee}$ to a unique alcove that has the origin as a vertex; i.e., to one of the alcoves in the $W$-orbit of $A_{\varnothing}$. The closure of this set of alcoves is the $W$-invariant convex polytope

$$
P_{\Phi}=\{\lambda \in V:-1 \leqslant\langle\lambda, \beta\rangle \leqslant 1 \text { for all } \beta \in \Phi\},
$$

and the Steinberg torus is obtained by identifying the maximal opposite faces of $P_{\Phi}$.

Example 2.2 The Steinberg torus for $\widetilde{A}_{2}$ is a hexagon with opposite sides identified, decomposed into six triangles, nine edges, and three vertices. See Figure 1 It has flag f-polynomial

$$
f\left(\Sigma_{T}\left(\widetilde{A}_{2}\right) ; t_{0}, t_{1}, t_{2}\right)=1+t_{0}+t_{1}+t_{2}+3 t_{0} t_{1}+3 t_{0} t_{2}+3 t_{1} t_{2}+6 t_{0} t_{1} t_{2} .
$$

Using (2.2) to compute the flag h-polynomial, we find

$$
h\left(\Sigma_{T}\left(\widetilde{A}_{2}\right) ; t_{0}, t_{1}, t_{2}\right)=1+2 t_{0} t_{1}+2 t_{0} t_{2}+2 t_{1} t_{2}-t_{0} t_{1} t_{2} .
$$


On the other hand, the reduced Steinberg torus lacks the empty face, so its flag $f$-polynomial omits the constant term and we find

$$
h\left(\Sigma_{T}^{\prime}\left(\widetilde{A}_{2}\right) ; t_{0}, t_{1}, t_{2}\right)=t_{0}+t_{1}+t_{2}+t_{0} t_{1}+t_{0} t_{2}+t_{1} t_{2} .
$$

Specializing, we see that the reduced Steinberg torus has ordinary $f$-polynomial $3 t+9 t^{2}+6 t^{3}$, and ordinary h-polynomial $3 t+3 t^{2}$.

Example 2.3 The Steinberg torus for $\widetilde{C}_{2}$ (or the isomorphic $\widetilde{B}_{2}$ ) is a square with opposite sides identified, decomposed into eight triangles, twelve edges, and four vertices as in Figure 1] The reduced Steinberg torus has flag $f$-polynomial

$$
f\left(\Sigma_{T}^{\prime}\left(\widetilde{C}_{2}\right) ; t_{0}, t_{1}, t_{2}\right)=t_{0}+t_{1}+2 t_{2}+4 t_{0} t_{1}+4 t_{0} t_{2}+4 t_{1} t_{2}+8 t_{0} t_{1} t_{2},
$$

and (again via 2.2) flag h-polynomial

$$
h\left(\Sigma_{T}^{\prime}\left(\widetilde{C}_{2}\right) ; t_{0}, t_{1}, t_{2}\right)=t_{0}+t_{1}+2 t_{2}+2 t_{0} t_{1}+t_{0} t_{2}+t_{1} t_{2} .
$$

As in the previous example, it is easy to check that the ordinary and flag h-polynomials of the unreduced Steinberg torus have (some) negative coefficients.

\subsection{Affine descents}

We define a root $\beta$ to be negative with respect to $w \in W$ if $w \beta<0$. The positive roots that are negative with respect to $w$ are known as inversions. If $\ell(w)$ denotes the minimum length of an expression for $w$ as a product of simple reflections, then $\beta$ is negative with respect to $w$ if $\ell\left(w s_{\beta}\right)<\ell(w)$ (for $\left.\beta>0\right)$ or $\ell\left(w s_{\beta}\right)>\ell(w)$ (for $\left.\beta<0\right)$.

A simple root that is negative with respect to $w$ is said to be a (right) descent, and the descent set of $w$, denoted $D(w)$, records the corresponding set of indices. Thus,

$$
D(w)=\left\{j \in[n]: w \alpha_{j}<0\right\}=\left\{j \in[n]: \ell\left(w s_{j}\right)<\ell(w)\right\} .
$$

We let $d(w):=|D(w)|$ denote the number of descents in $w$.

As noted in the introduction, the $W$-Eulerian polynomial is the $h$-polynomial of the Coxeter complex $\Sigma(W)$. That is,

$$
W(t)=\sum_{w \in W} t^{d(w)}=h(\Sigma(W) ; t)
$$

More generally, the generating function for descent sets; namely,

$$
W\left(t_{1}, \ldots, t_{n}\right):=\sum_{w \in W} \prod_{j \in D(w)} t_{j}
$$

is the flag $h$-polynomial of $\Sigma(W)$ (e.g., see the discussion at the end of Section III.4 in (21)).

Extending these concepts, set $\alpha_{0}:=-\widetilde{\alpha}$ (the lowest root), and let $s_{0}=s_{\widetilde{\alpha}}$ denote the corresponding reflection in $W$. We define the affine descent set of $w$, denoted $\widetilde{D}(w)$, to be the set indices of roots in $\Delta_{0}:=\Delta \cup\left\{\alpha_{0}\right\}$ that are negative with respect to $w$. Thus,

$$
\widetilde{D}(w)=\left\{j \in[0, n]: w \alpha_{j}<0\right\}=\left\{\begin{array}{cl}
D(w) \cup\{0\} & \text { if } \ell\left(w s_{0}\right)>\ell(w), \\
D(w) & \text { if } \ell\left(w s_{0}\right)<\ell(w) .
\end{array}\right.
$$


We let $\widetilde{d}(w):=|\widetilde{D}(w)|$ denote the number of affine descents in $w$.

Note that only the identity element of $W$ has an empty descent set (but has an affine descent at 0 ), and only the longest element $w_{0}$ has a full descent set (i.e., $D\left(w_{0}\right)=[n]$ ) but does not have an affine descent at 0 . Thus $1 \leqslant \widetilde{d}(w) \leqslant n$ for all $w \in W$.

\section{Affine Eulerian polynomials}

We let $\widetilde{W}\left(t_{0}, \ldots, t_{n}\right)$ and $\widetilde{W}(t)$ denote the respective generating functions for affine descent sets and numbers of affine descent sets; i.e.,

$$
\begin{aligned}
\widetilde{W}\left(t_{0}, \ldots, t_{n}\right) & :=\sum_{w \in W} \prod_{j \in \widetilde{D}(w)} t_{j}, \\
\widetilde{W}(t):=\widetilde{W}(t, \ldots, t) & =\sum_{w \in W} t^{\widetilde{d}(w)} .
\end{aligned}
$$

We refer to these as multivariate and univariate affine Eulerian polynomials.

Theorem 3.1 If $\widetilde{W}$ is an irreducible affine Weyl group, then the flag h-polynomial of the corresponding reduced Steinberg torus is the multivariate $\widetilde{W}$-Eulerian polynomial; i.e.,

$$
h\left(\Sigma_{T}^{\prime}(\widetilde{W}) ; t_{0}, \ldots, t_{n}\right)=\widetilde{W}\left(t_{0}, \ldots, t_{n}\right) .
$$

In particular, for all $J \subseteq[0, n]$, we have

$$
\begin{aligned}
& f_{J}\left(\Sigma_{T}^{\prime}(\widetilde{W})\right)=|\{w \in W: \widetilde{D}(w) \subseteq J\}|, \\
& h_{J}\left(\Sigma_{T}^{\prime}(\widetilde{W})\right)=|\{w \in W: \widetilde{D}(w)=J\}| .
\end{aligned}
$$

Furthermore,

$$
\widetilde{W}\left(t_{0}, \ldots, t_{n}\right)=\sum_{J \subsetneq[0, n]} \frac{|W|}{\left|W_{J}\right|} \prod_{j \in J}\left(1-t_{j}\right) \prod_{j \notin J} t_{j},
$$

where $W_{J}$ denotes the (not necessarily parabolic) subgroup of $W$ generated by $\left\{s_{j}: j \in J\right\}$.

Of course it follows immediately that the ordinary $h$-polynomial of the reduced Steinberg torus is the corresponding univariate affine Eulerian polynomial; i.e.,

$$
h\left(\Sigma_{T}^{\prime}(\widetilde{W}) ; t\right)=\widetilde{W}(t)=\sum_{w \in W} t^{\widetilde{d}(w)}
$$

By considering the effect on descent sets of left multiplication by the longest element $w_{0}$, we get symmetry as an easy corollary.

Corollary 3.2 The flag h-vector of the reduced Steinberg torus $\Sigma_{T}^{\prime}(\widetilde{W})$ satisfies the generalized DehnSommerville equations in the sense that, for all $J \subseteq[0, n]$, we have

$$
h_{J}\left(\Sigma_{T}^{\prime}(\widetilde{W})\right)=h_{J^{c}}\left(\Sigma_{T}^{\prime}(\widetilde{W})\right) .
$$

In particular, the $\widetilde{W}$-Eulerian polynomial is symmetric: $\widetilde{W}(t)=t^{n+1} \widetilde{W}(1 / t)$. 


\begin{tabular}{l|c}
$W$ & $\widetilde{W}(t)$ \\
\hline$B_{3}$ & $10 t+28 t^{2}+10 t^{3}$ \\
\hline$B_{4}$ & $24 t+168 t^{2}+168 t^{3}+24 t^{4}$ \\
\hline$B_{5}$ & $116 t+4452 t^{2}+18472 t^{3}+18472 t^{4}+4452 t^{5}+116 t^{6}$ \\
\hline$B_{6}$ & $242 t+20612 t^{2}+157294 t^{3}+288824 t^{4}+157294 t^{5}+20612 t^{6}+242 t^{7}$ \\
\hline$B_{7}$ & $16 t+80 t^{2}+80 t^{3}+16 t^{4}$ \\
\hline \hline$D_{4}$ & $104 t+2568 t^{2}+8848 t^{3}+8848 t^{4}+2568 t^{5}+104 t^{6}$ \\
\hline$D_{5}$ & $351 t+5427 t^{2}+20142 t^{3}+20142 t^{4}+5427 t^{5}+351 t^{6}$ \\
\hline$D_{6}$ & $+13192 t^{2}+79580 t^{3}+136560 t^{4}+79580 t^{5}+13192 t^{6}+228 t^{7}$ \\
\hline$D_{7}$ & $72 t+504 t^{2}+504 t^{3}+72 t^{4}$ \\
\hline \hline$E_{6}$ & $6 t+6 t^{2}$ \\
\hline$E_{7}$ & $4064 t+115728 t^{2}+710112 t^{3}+1243232 t^{4}+710112 t^{5}+115728 t^{6}+4064 t^{7}$ \\
\hline$E_{8}$ & $157200 t+9253680 t^{2}+87417360 t^{3}+251536560 t^{4}$ \\
\hline$F_{4}$ & $+251536560 t^{5}+87417360 t^{6}+9253680 t^{7}+157200 t^{8}$ \\
\hline$G_{2}$ & \\
\hline
\end{tabular}

Tab. 1: Some affine Eulerian polynomials.

Remark 3.3 The unreduced Steinberg torus $\Sigma_{T}(\widetilde{W})$ has nearly the same flag f-vector as its reduced counterpart, the only difference being $f_{\varnothing}\left(\Sigma_{T}(\widetilde{W})\right)=1$ in place of $f_{\varnothing}\left(\Sigma_{T}^{\prime}(\widetilde{W})\right)=0$. However, as we noted in Example 2.2 the h-polynomial need not have symmetric or nonnegative coefficients in the unreduced case, and is therefore of less interest.

The following lemma is the key to our proof of Theorem 3.1, which can be found in (8).

Lemma 3.4 If $\left\{\beta_{i}: i \in I\right\}$ is a set of simple roots for a reflection subgroup $W^{\prime}$ of $W$, then every coset in $W / W^{\prime}$ has a unique member $w$ such that $w \beta_{i}>0$ for all $i \in I$.

Remark 3.5 In the above lemma, it is interesting to note that by choosing the simple roots of $W^{\prime}$ so that they are positive relative to $\Phi$, one may deduce that every coset of every reflection subgroup of $W$ has a unique element of minimum length. This is a familiar fact for parabolic subgroups, but the less familiar general case also follows from work of Dyer (see Corollary 3.4 of (9)).

It is easy to compute the affine Eulerian polynomials for the groups of low rank via 3.6. Some examples, including all of the exceptional groups, are listed in Table 1. 
Remark 3.6 Given $J \subsetneq[0, n]$, it follows from Lemma 3.4 that each coset in $W / W_{J}$ has a unique representative $w$ such that $\widetilde{D}(w) \cap J=\varnothing$. Thus each cell of the reduced Steinberg torus has the form $F(w, J)=w A_{J}+\mathbb{Z} \Phi^{\vee}$ for some unique pair $(w, J)$ with $\widetilde{D}(w) \cap J=\varnothing$. Moreover, the cells of the form $F(w, *)$ are precisely the cells in the closure of $F(w, \varnothing)$ that have on their boundary the unique cell with color-set $\widetilde{D}(w)$; namely, $F\left(w, \widetilde{D}(w)^{c}\right)$. Thus the reduced Steinberg torus is "partitionable" in the sense defined in Section III.2 of (21).

\section{Real roots, $\gamma$-vectors, and unimodality}

The following is a companion to Brenti's conjecture (2) that the roots of all (ordinary) Eulerian polynomials $W(t)$ are real.

Conjecture 4.1 The roots of all affine Eulerian polynomials $\widetilde{W}(t)$ are real.

To complete a proof of this conjecture, we claim that it suffices to consider only the groups $\widetilde{B}_{n}$ and $\widetilde{D}_{n}$. Indeed, it follows from observations of Fulman $(11,12)$ and Petersen $(18)$ that $\widetilde{A}_{n}(t)$ and $\widetilde{C}_{n}(t)$ are both multiples of $A_{n-1}(t)$ (see also Section 5 below). Thus the conjecture for $\widetilde{A}_{n}$ and $\widetilde{C}_{n}$ follows from the fact that all roots of the classical Eulerian polynomials are known to be real (14). Furthermore, using the data in Table 1 , it is easy to check that the conjecture holds for the exceptional groups.

To collect supporting evidence for the remaining groups $\widetilde{B}_{n}$ and $\widetilde{D}_{n}$, we have determined explicit exponential generating functions for the corresponding affine Eulerian polynomials (see Proposition 6.2 below), and used these to verify the conjecture for $n \leqslant 100$. In a similar way, we have also confirmed that all roots of $D_{n}(t)$ are real (the only remaining open case of Brenti's conjecture) for $n \leqslant 100$.

A further supporting result involves $\gamma$-vectors in the sense of Brändén (1) and Gal (13). To explain, consider a polynomial satisfying $h(t)=t^{m} h(1 / t)$. It is clear that such a polynomial has a unique expansion of the form

$$
h(t)=\sum_{0 \leqslant i \leqslant m / 2} \gamma_{i} t^{i}(1+t)^{m-2 i} .
$$

We call $\left(\gamma_{0}, \gamma_{1}, \ldots\right)$ the $\gamma$-vector of $h(t)$.

It is elementary to show that if $h(t)$ has symmetric, nonnegative coefficients and all real roots, then it has a nonnegative $\gamma$-vector (see Lemma 4.1 of (1) or Section 1.4 of (26)).

Recall that $\widetilde{W}(t)$ is symmetric (Corollary 3.2 , so it has a $\gamma$-vector. Given that we know Conjecture 4.1 holds for $\widetilde{A}_{n}, \widetilde{C}_{n}$, and the exceptional affine Weyl groups, it follows that $\widetilde{W}(t)$ is $\gamma$-nonnegative in each of these cases. For $\widetilde{B}_{n}$ and $\widetilde{D}_{n}$ we provide explicit combinatorial descriptions of the $\gamma$-vector in the following section. Thus we have the following.

Theorem 4.2 The affine Eulerian polynomials $\widetilde{W}(t)$ have nonnegative $\gamma$-vectors.

It would be interesting to have a conceptual (case-free) proof of this result.

Any polynomial with a nonnegative $\gamma$-vector has unimodal coefficients. Hence,

Corollary 4.3 The affine Eulerian polynomials have unimodal coefficients.

We remark that the $\gamma$-vectors of the Eulerian polynomials $W(t)$ are also known to be nonnegative, but the only existing proofs to date are case-by-case $(\underline{6}, 26)$. 


\section{Combinatorial expansions and $\gamma$-nonnegativity}

In this section, we provide combinatorial expansions for the ordinary and affine Eulerian polynomials for the four infinite families of irreducible Weyl groups. Each identity is obtained by specializing certain formulas for the multivariate affine Eulerian polynomials (not included here; see Section 5 of $(\underline{8})$ ). As corollaries, we will deduce nonnegativity of the $\gamma$-vectors for these polynomials. Proofs and other typespecific details have been omitted, but can be found in (8).

It will be convenient for what follows to use two conventions for counting peaks in a permutation $u \in S_{n}$; namely,

$$
\begin{aligned}
\operatorname{lpk}(u) & :=\left|\left\{i \in[1, n-1]: u_{i-1}<u_{i}>u_{i+1}\right\}\right|, \\
\operatorname{epk}(u) & :=\left|\left\{i \in[1, n]: u_{i-1}<u_{i}>u_{i+1}\right\}\right|
\end{aligned}
$$

with the convention $u_{0}=u_{n+1}=0$. We refer to these quantities as the number of left and extended peaks in $u$, respectively.

Proposition 5.1 We have,

$$
\begin{aligned}
\widetilde{A}_{n}(t)=(n+1) t A_{n-1}(t) & =\frac{n+1}{2^{n+1}} \sum_{u \in S_{n}}(4 t)^{\operatorname{epk}(u)}(1+t)^{n+1-2 \operatorname{epk}(u)}, \\
\widetilde{C}_{n}(t)=2^{n} t A_{n-1}(t) & =\frac{1}{2} \sum_{u \in S_{n}}(4 t)^{\operatorname{epk}(u)}(1+t)^{n+1-2 \operatorname{epk}(u)}, \\
C_{n}(t)=B_{n}(t) & =\sum_{u \in S_{n}}(4 t)^{\operatorname{lpk}(u)}(1+t)^{n-2 \operatorname{lpk}(u)}, \\
\widetilde{B}_{n}(t) & =\sum_{u \in S_{n}} \phi(u)(4 t)^{\operatorname{epk}(u)}(1+t)^{n+1-2 \operatorname{epk}(u),} \\
D_{n}(t) & =\sum_{u \in S_{n}} \phi\left(u^{*}\right)(4 t)^{\operatorname{lpk}(u)}(1+t)^{n-2 \operatorname{lpk}(u)}, \\
\widetilde{D}_{n}(t) & =\sum_{u \in S_{n}} \phi(u) \phi\left(u^{*}\right)(4 t)^{\operatorname{epk}(u)}(1+t)^{n+1-2 \operatorname{epk}(u),}
\end{aligned}
$$

where

$$
\phi(u):=\left\{\begin{array}{cl}
1 & \text { if } u_{n-2}>u_{n-1}>u_{n} \\
0 & \text { if } u_{n-2}>u_{n}>u_{n-1} \\
1 / 2 & \text { otherwise }
\end{array}\right.
$$

and $u^{*}:=u_{n} \cdots u_{2} u_{1}$.

Some of these identities are already known. That the affine Eulerian polynomials of types $A$ and $C$ are multiples of classical Eulerian polynomials (left hand sides of (5.1) and (5.2)) was noted previously by Fulman $(11 ; 12)$ and Petersen (18). The right hand side of $(5.1)$ is equivalent to an identity due to Foata and Schützenberger (Théorème 5.6 of (10); see also Remark 4.8 of (25)), equation (5.3) is due to Petersen (Proposition 4.15 in (19)), and equation (5.5) is due to Stembridge (Corollary A.5 in (26); compare also Theorem 6.9 in (ㅁ) $)$. 


\section{Identities and generating functions}

Here we provide several unexpected identities (two new, one old) relating the ordinary and affine Eulerian polynomials. The proofs, which are omitted, are bijective and rely on the combinatorial expansions from the previous section.

Proposition 6.1 We have

$$
\begin{aligned}
2 \widetilde{C}_{n}(t) & =\widetilde{B}_{n}(t)+2 n t C_{n-1}(t), \\
\widetilde{B}_{n}(t) & =\widetilde{D}_{n}(t)+2 n t D_{n-1}(t), \\
B_{n}(t)=C_{n}(t) & =D_{n}(t)+n 2^{n-1} t A_{n-2}(t) .
\end{aligned}
$$

Equation (6.3) is due to Stembridge (set $l=0$ in (24, Lemma 9.1)).

From Proposition 6.1 it is easy (given the known generating functions for ordinary Eulerian polynomials; see (7. p. 244), Theorem 3.4 and Corollary 4.9 in (2)) to construct generating functions for each infinite family of affine Eulerian polynomials.

Proposition 6.2 We have

$$
\begin{gathered}
\widetilde{A}(t, z)=z+\sum_{n \geqslant 2} \widetilde{A}_{n-1}(t) \frac{z^{n}}{n !}=\frac{z(1-t)}{1-t e^{z(1-t)}}, \\
\widetilde{C}(t, z)=1+\sum_{n \geqslant 1} \widetilde{C}_{n}(t) \frac{z^{n}}{n !}=\frac{1-t}{1-t e^{2 z(1-t)}}, \\
\widetilde{B}(t, z)=2+2 t z+\sum_{n \geqslant 2} \widetilde{B}_{n}(t) \frac{z^{n}}{n !}=\frac{2(1-t)\left(1-t z e^{z(1-t)}\right)}{1-t e^{2 z(1-t)}}, \\
\widetilde{D}(t, z)=2+4 t \frac{z^{2}}{2}+\sum_{n \geqslant 3} \widetilde{D}_{n}(t) \frac{z^{n}}{n !}=\frac{2(1-t)\left(1+t z^{2}-2 t z e^{z(1-t)}\right)}{1-t e^{2 z(1-t)}} .
\end{gathered}
$$

\section{References}

[1] P. Brändén, Sign-graded posets, unimodality of $W$-polynomials and the Charney-Davis conjecture, Electron. J. Combin. 11 (2004/06), Research Paper 9, 15pp.

[2] F. Brenti, $q$-Eulerian polynomials arising from Coxeter groups, European J. Combin. 15 (1994), $417-441$.

[3] P. Cellini, A general commutative descent algebra, J. Algebra 175 (1995), 990-1014.

[4] P. Cellini, A general commutative descent algebra II: The case $C_{n}$, J. Algebra 175 (1995), 10151026.

[5] P. Cellini, Cyclic eulerian elements, European. J. Combin. 19 (1998), 545-552.

[6] C.-O. Chow, On certain combinatorial expansions of the Eulerian polynomials, Adv. in Appl. Math., to appear. 
[7] L. Comtet, "Advanced Combinatorics," Reidel, Dordrecht, 1974.

[8] K. Dilks, T. K. Petersen, and J. R. Stembridge, Affine descents and the Steinberg torus, arXiv:0709.4291.

[9] M. Dyer, Reflection subgroups of Coxeter systems, J. Algebra 135 (1990), 57-73.

[10] D. Foata and M.-P. Schützenberger, "Théorie géométrique des polynômes eulériens," Lecture Notes in Mathematics, Vol. 138, Springer-Verlag, Berlin, 1970.

[11] J. Fulman, Affine shuffles, shuffles with cuts, the Whitehouse module, and patience sorting, J. Algebra 231 (2000), 614-639.

[12] J. Fulman, Applications of the Brauer complex: card shuffling, permutation statistics, and dynamical systems, J. Algebra 243 (2001), 96-122.

[13] S. R. Gal, Real root conjecture fails for five- and higher-dimensional spheres, Discrete Comput. Geom. 34 (2005), 269-284.

[14] L. H. Harper, Stirling behavior is asymptotically normal, Ann. Math. Statist. 38 (1967), 410-414.

[15] J. E. Humphreys, "Reflection groups and Coxeter groups," Cambridge Univ. Press, Cambridge, 1990.

[16] T. Lam and A. Postnikov, Alcoved polytopes II, in preparation.

[17] I. Novik and E. Swartz, Socles of Buchsbaum modules, complexes and posets, preprint.

[18] T. K. Petersen, Cyclic descents and P-partitions, J. Algebraic Combin. 22 (2005), 343-375.

[19] T. K. Petersen, Enriched P-partitions and peak algebras, Adv. Math. 209 (2007), 561-610.

[20] A. Postnikov, V. Reiner, and L. Williams, Faces of generalized permutohedra, preprint.

[21] R. P. Stanley, "Combinatorics and Commutative Algebra" (2nd ed.), Birkhäuser, Boston, 1996.

[22] R. P. Stanley, $f$-vectors and $h$-vectors of simplicial posets, J. Pure Appl. Algebra 71 (1991), 319331.

[23] R. Steinberg, "Endomorphisms of linear algebraic groups," Mem. Amer. Math. Soc. No. 80 (1968), American Mathematical Society, Providence, RI.

[24] J. R. Stembridge, Some permutation representations of Weyl groups associated with the cohomology of toric varieties, Adv. Math. 106 (1994), 244-301.

[25] J. R. Stembridge, Enriched P-partitions, Trans. Amer. Math. Soc. 349 (1997), 763-788.

[26] J. R. Stembridge, Coxeter cones and their $h$-vectors, Adv. Math., to appear.

[27] E. Swartz, personal communication. 
